Kinga Wysienska-Di Carlo

Zbigniew Karpinski

Institute of Philosophy and Sociology

Polish Academy of Sciences

\title{
Just gender pay gap and \\ motherhood penalty - results of two vignette experiments. A research note
}

Acknowledgements:

Research reported in this paper was supported by a grant from National Science Centre, Poland (Grant No. 2015/19/B/HS6/03169).

A research note prepared for the 2019 Motherhood Penalty Workshop at the Institute of Philosophy and Sociology of the Polish Academy of Sciences.

\section{Background}

It is often argued (e.g., Jasso and Webster 1997; Auspurg et al. 2017) that one reason why gender inequality persists across time and space is because it is legitimized. In this paper, we explain and test when (i.e., under what conditions) and what amount of gender inequality is legitimized. We are especially interested in parental status effects.

We jointly apply three theories of the expectations states program: status characteristics theory; double-standards theory; and reward-expectations theory. Put simply, the theories we use postulate that if gender and motherhood are status characteristics, there are certain expectations attached to them. These expectations are higher for men vs. women and fathers vs. mothers, especially in a job context. These 
expectations, in turn, affect rewards and assessments of just rewards. Moreover, the consistency between certain characteristics and outcomes matters in the eyes of those evaluating just rewards.

Based on these theories we derived a set of hypotheses about the relationship between gender, motherhood, and job related attributes, and how much inequality is legitimized and under what conditions. In order to test our hypotheses, we use a vignette (multifactorial) design. An important advantage of using vignettes in sociological research is that they allow for obtaining judgments from respondents concerning combinations of characteristics that occur rather rarely in the population (Heise 2010, chap. 4; Wallander 2009). Also, to study status inconsistency effects on perceptions of justice of earnings, one needs a reasonably large number of status-inconsistent situations in order to be able to compare them systematically with status-consistent ones. Thus, the result of the test depends, among other things, on the relative frequency of status inconsistency in the population under study. In a vignette study, this is of little concern because the combination of characteristics assigned to a vignette is subject to explicit manipulation.

Our findings indicate that there is very little legitimation of gender inequalities in pay in Poland. In most situations the gap is less than 5\% and statistically insignificant. The magnitude of accepted inequality between genders and parents varies by occupational prestige and experience. That is, there is virtually no accepted inequality in medium prestige occupations and slightly higher accepted inequality in high prestige occupations, as opposed to low prestige occupations. Additional work experience matters only for mothers of three or more children, who also experience the highest (perceived as just) motherhood penalty in high prestige occupations.

\section{Motherhood and fatherhood as status characteristics - theory}

This project relies on the application of various concepts stemming from the expectation states theory program. It is an ongoing research program that accounts for various processes responsible for the emergence and stability of status structures. More importantly, it also explains how status beliefs and status structures are translated into an 
unequal distribution of socially valued resources, both material (e.g., remuneration) and non-material (recognition, deference, influence). In other words, various theories within the program explicate how beliefs regarding various social attributes emerge and affect micro-interactions, and how they aggregate to macro consequences. Those macro consequences, in turn, reinforce beliefs and solidify social inequalities structure.

Expectation states theories, of course, apply only if certain conditions are met (socalled scope conditions). Those conditions include collective and task orientation. Collective orientation refers to contexts in which actors need to take each other (or generalized-other opinions) into account in order to achieve success at the task at hand or to solve a problem (task orientation). Work-related situations meet these conditions. Hiring, evaluating the right amount of salary, assessing someone's competence, etc., all require comparing an applicant (or a profile in the vignette) to another applicant (or a profile in the vignette), or to some ideal notion of a "good" applicant (or a profile in a vignette). Assessing skills and appropriate rewards is also a valued task. After all, if there is a discrepancy between what is and what should be, the consequences will spill over not only to those in the assessment, but also to observers of the situation, other situations, etc.

Expectation states theory's main argument proposes that people form expectations based on various kinds of information in order to form a mental picture of who is who and who can do what, in order to know how to act in a situation or during a social interaction (that meets specified earlier scope conditions). Expectations as such are unobservable and often unconscious. They are formed based on salient (ascribed or achieved) attributes (social categories) of persons involved in a given context or cognitively available to the individual (social norms, opinions of significant others), previous actions or performance outcomes, existing distribution of rewards, and so on.

The main sources of high or low expectations are social attributes, such as gender or skin color, that visibly differentiate between actors and are linked to widely shared beliefs about certain types or levels of skill associated with them. Such attributes are referred to as status characteristics and defined in Status Characteristics Theory (Berger, Webster 2006; Ridgeway 2001, 2014). More specifically, the notion refers to categorical distinctions that give rise to status beliefs that accord more competence, esteem, and general social worth to one 'state' of the underlying characteristic than to other state(s) (Ridgeway 2006). SCT theory distinguishes two types of status characteristics, specific 
and diffuse, based on the range of situations in which each type becomes relevant. Specific status characteristics are particular abilities that produce well-defined performance expectations relevant to a particular type of task (e.g., years of experience at a given job). A characteristic is diffuse if: (1) the characteristic has two or more states that the actors evaluate differently; (2) there is a set of one or more specific characteristics associated with each state of the diffuse characteristic; and (3) the actor associates a general expectation with each status state. Any characteristic that differentiates between actors, or that is believed to be relevant to the task at hand, will become salient, unless demonstrated to be dissociated from the task (according to the salience and burden of proof assumptions of the theory) (Berger and Webster 2006). Which distinctions carry status value and their specific contents may vary across cultures, but gender, race/ethnicity, age and occupation are almost universally linked to status beliefs.

A specific characteristic is directly relevant to a particular task, whereas a diffuse characteristic is linked to many tasks, although indirectly. Therefore, the weight of each type of characteristic on aggregate performance expectations may differ. When this is the case, status characteristics that are directly relevant to the task may have more weight in forming aggregate expectations than do status characteristics that are not directly relevant.

Motherhood is argued to be a status characteristic because there is a set of diverse attributes that are evaluated in a particular (devalued) way when linked to a mother than when linked to a non-mother.

However, for motherhood to be a diffuse status characteristic, being a mother needs to represent a level of a distinction that has at least one clearly delineated "comparison" level. Ridgeway and Correll (2004) argue that this level is being a nonmother - i.e. a childless person of any gender or a father who is not the primary caregiver (in this view, motherhood is not exclusive to females - a male parent who takes the role of a primary caregiver becomes a "mother").

That would mean, however, that any relevant outcomes (evaluations, rewards, employment decisions, etc.) would have to be compared between "mothers" and everybody else. In contrast, we argue that if a male becomes a primary caregiver for a child, he does not necessarily become a "mother." Expectations towards and evaluations 
of a male parent are different from those of a female parent regardless of level of involvement in caregiving/parenting (Kmec et al. 2014). In other words, we argue that both motherhood and fatherhood are status characteristics, that they cannot be simplified to "being a parent" vs. "being childless," and that they are gender specific - i.e., that they interact with expectations regarding gender as a status characteristic.

In most social situations, actors are differentiated by two or more status characteristics, which are often allocated inconsistently — i.e., actors possess a high level of one characteristic, but a low level of another. Sociologists have long been interested in the effects of status inconsistency on judgments, expectations, and behavior. For instance, there is a large literature focused on how status inconsistency affects expectations as to fair or deserved job income (e.g., Słomczynski and Wesołowski, 2001). According to SCT, in such situations, actors forming their performance expectations combine all the status information that is salient or relevant to the task. According to the principle of organized subsets, all information leading to positive performance expectations is combined by the actor into a subset to determine its positive value, while all information leading to negative performance expectations is combined into a subset to determine its negative value.

As mentioned above, this combining process takes into account that status distinctions differ in terms of their relevance to the immediate task. Status information processing also conforms to the attenuation principle, according to which each additional piece of consistently valued status information will have a smaller marginal effect on one's status advantage or disadvantage. As attenuation occurs within each (positive and negative) subset, the subset with the least elements will be most susceptible to additional information. For instance, a man with a university degree has a status advantage over a woman with the same credential. Yet, suppose that that same man is a surgeon and the woman is a pediatrician. In this case, his status advantage will increase, but this increase will be smaller than that resulting from the activation of gender alone. If the woman, however, is a professor of pediatrics and the man is only a hospital surgeon, his advantage will decrease significantly (Berger et al. 1992).

The aggregated expectations for a given actor are determined by summing the values of the positive and negative subsets. The actor's expectation advantage (or 
disadvantage) relative to another actor is equal to his or her aggregated expectation minus that of the other actor (Berger and Webster 2006).

Status characteristics not only lead to biased evaluations and divergent expectations (which subsequently guide decisions and behaviors towards persons with particular levels of status distinctions), but they also result in different standards being used to determine whether given performance constitutes proof of ability and competence level. According to Double Standards Theory (Foschi 1996), a stricter standard to prove competence will be applied to a person with lower status, while a more lenient standard will be used for a person with higher status. This is because, when high status individuals perform well, the assessor's expectations are met, and there is no cognitive inconsistency to resolve. When low status individuals perform well, their good performance appears inconsistent with what was anticipated, thus tending to require further scrutiny on the part of the assessor (Foschi 2000).

Status characteristics also play a major role in the formation of reward expectations. According to Reward Expectations Theory (Berger et al. 1985), sets of socially validated beliefs (referential structures) are held in common by actors. These beliefs describe what is thought to be the usual association between a valued characteristic and levels of rewards. The theory distinguishes three types of referential structures - categorical, ability, and outcome structures - and describes conditions under which each is activated. The differences between the types of referential structures pertain to the type of information that is linked with reward levels. In the case of categorical referential structures, the information in question is membership in broad social categories, such as gender or motherhood. In the case of ability-based referential structures, the relevant information is a person's ability to perform a task well, or his or her ability to contribute to the group's goal. Finally, outcome-based referential structures link rewards to what one has actually accomplished or achieved (Berger et al. 1985).

These beliefs, when activated, influence the allocation of rewards, which itself is influenced by status situations. There are situations where only performance-relevant skills matter, situations where capacities, accomplishments, and status categories matter, and situations where only status category matters.

A basic corollary of this theory is that if multiple referential beliefs are activated in a situation, actors will combine these structures in forming their reward expectations. 
Similarly, if multiple status distinctions exist in a situation, actors will combine information from these characteristics.

\section{Vignette population and vignette samples}

\section{Study 1}

Each participant in our survey was assigned a sample of vignettes. The samples were drawn at random from a population of vignettes. Crossing levels of four characteristics, gender, age, occupation, and parenthood status (or the number of children) created the population of vignettes. Age varied between 30 and 65 in 5-years increments.

Occupations were classified into three categories of prestige: high, medium, and low. The basis of that classification was a recent scale of occupational prestige rooted in the Polish Sociological Classification of Occupations (Domanski, Słomczynski, and Sawinski 2009). More specifically, the scale assigns a score between 0 and 100 to each occupation listed in the classification, with higher scores indicating higher prestige. For the purpose of the our study, we grouped the occupations into three broad classes, so that the high category comprises occupations with a score of 70 or higher, the low category -occupations with a score below 40 , and the middle category includes occupations with scores ranging from 40 to 69 . For each level of prestige, seven occupations were selected. ${ }^{1}$ Because we wanted vignette samples assigned to respondents to cover the full spectrum of occupational prestige, we used stratified sampling to select a sample for each respondent, with each occupation being a separate stratum. For the number of children, there were three levels: no children, one child, and two or more children.

This means that with 2 gender categories, 8 age levels, 21 occupational titles, and 3 levels of the parenthood status, we had a total of 1,008 combinations. Removing

\footnotetext{
${ }^{1}$ The occupations used in the vignettes and their corresponding prestige scores (in the brackets) are as follows: professor of early education (97.3), anesthesiologist (87.8), actor in a theatre (86.9), radio news presenter (81.2), plane pilot (80.6), IT security specialist (77.5), artistic director in an ad agency (72.7), animal technician (66.1), librarian (61.3), train dispatcher (60.9), nurse (55.9), customs officer (54.9), real estate agent (51.4), accountant (50.9), cashier in a supermarket (27.6), mailperson (27.5), waitperson (25.7), hairdresser (21.2), telemarketer (18.0), janitor (10.4), cloakroom attendant (8.6).
} 
combinations that are logically improbable reduced the total number of available vignettes to $996 .^{2}$

Three final remarks concerning the vignettes are in order. First, apart from the four characteristics listed above, individual vignettes also included information about job experience. However, that information was added to the vignettes after the vignette population was created. Moreover, it was closely associated with age. ${ }^{3}$ In fact, the correlation between age and job experience in the vignette population was 0.99. Second, for the vignettes in the "two or more children" category, we actually distinguished between individuals with 2, 3, or 4 children. That is, vignettes in that category were assigned 2 children with probability $0.50,3$ children with probability 0.35 , and 4 children with probability 0.15 when making individual vignettes. This was done to make our vignettes look more "realistic", as we thought that describing individuals as having "two or more children" might appear somewhat artificial to our respondents.

Also, it is important to keep in mind that for vignettes in the "no children" category, no information about the number of children was provided. That is, vignettes in this category were not presented as having no children; instead, no information about children was included in these vignettes. This might have some effect on how our respondents perceived these vignettes, because they could have made implicit assumptions about their parenthood status based on their other characteristics.

Finally, the vignettes were preceded by a general description of the task and "rewardees" population. That is, we explained that all "rewardees" are full-time employees, live in a medium size town, have always been positively evaluated by their supervisors, and were never unemployed. The purpose was to keep "outcome referential structure" constant as well as (indirectly) test statistical discrimination effects. To achieve this latter goal, we randomly assigned respondents to one of two versions of instructions. The second version included only information that job performance was always

\footnotetext{
${ }^{2}$ One of the occupations that we used in our vignettes was the professor of early education. Because it is unlikely to obtain professorship by the age of 40 in the Polish academic system, we decided to remove all the vignettes in which the job a professor was combined with an age younger than 40 .

${ }^{3}$ For each vignette in our vignette population, job experience was obtained by adding a small amount of random variation to a linear transformation of age, the transformation being age less the years spent in education.
} 
satisfactory. The type of instruction had no effect on the just pay gaps, so we do not include it in our analysis below.

From the vignette population, we drew samples of 21 vignettes for each respondent separately, as mentioned above. Because there were 996 unique vignettes and 2,001 participants in our study, each vignette was evaluated by more than one respondent. Further, to each vignette in a vignette sample we assigned a set of six amounts of earnings. These amounts were presented as referring to net monthly on-the-job earnings and they were drawn from a hypothetical distribution, the parameters of which closely resembled those of the actual distribution of earnings in Poland in 2016. Importantly, these amounts were sampled separately for each vignette and they were listed in random order. For each amount assigned to a vignette, we asked the respondent how "appropriate" he or she thought this amount would be for the person described in the vignette. Responses were coded on a scale ranging from -5 ("much too low") to 5 ("much too high") with 0 labeled as "appropriate". With 21 vignettes to evaluate, and 6 amounts of earnings assigned to each vignette, each respondent generated 126 evaluations of the justice of earnings.

\section{Study 2}

The design of our second study followed closely that of Study 1, with some modifications. First, we reduced the number of occupations at each prestige level from 7 to 5 . As a result, the size of the vignette samples assigned to each respondent was 15 , not $21 .^{4}$ This gave us a total of 720 combinations. However, as noted previously, some of the combinations were removed due to their being logically improbable, which reduced the number of vignettes to $702 .{ }^{5}$ Second, we created two populations of vignettes, which were identical in all respects except for the information about job experience. In the population that we simply labeled as "A", job experience was pretty much the same as in the vignette population from our first study, indicating that the individuals described in the vignettes worked without break after completing their education. In the population

\footnotetext{
${ }^{4}$ The following occupational titles were not used to create vignettes in Study 2: actor in a theatre, radio news presenter, animal technician, customs officer, telemarketer, and cloakroom attendant.

${ }^{5}$ Apart from excluding vignettes in which "professor of early education" was combined with an age below 40, we also excluded, for similar reasons, ones in which the occupation of anesthesiologist was combined with an age below 30 .
} 
that we labeled as "B", the job experience was about 5 years shorter than in Population A, in order to allow for possible career breaks. Importantly, participants in Study 2 were assigned vignettes from one population only. Thus, the vignette population is a betweensubject variable.

\section{Respondent samples}

\section{Study 1}

The vignette survey was administered to a sample of registered members of an Internet panel run by one of the leading marketing and opinion research companies in Poland in 2017 ( $\mathrm{n}=2,001$ respondents). The sample was not random, but it was designed to match the composition of the Polish working population in terms of age, gender, education, and geographical location. Approximately half of our respondents were female, with a mean age of 41.4 years.

With 2,001 study participants, and 126 observations generated by a single respondent, the total number of justice evaluations in the resulting dataset is 252,126. However, the size of the dataset that we used in the analyses is much smaller. First, preliminary inspection of the data revealed that some respondents gave "illogical" responses when viewing some of the vignettes. We classified a set of responses as illogical if: (a) the respondent gave identical evaluations to all amounts assigned to the vignette; or $(b)$ there were some contradictions in how the respondent evaluated amounts assigned to the vignette. As regards the latter, suppose the amounts in question are 2,700, 8,900, and 4,500 in Polish currency and the respondent's evaluations are 1, -3 , and 2, respectively. As we can see, the smallest of the three amounts is seen by the respondents as much more "appropriate" than the largest amount, but then the smallest amount is less appropriate than the medium amount.

Second, and more importantly, we used the six evaluations of justice in each vignette-respondent combination to estimate a just amount of earnings for this vignette in the eyes of the respondent. In doing so, we followed the procedure developed by Jasso and Webster (1999). That is, even if there were no inconsistencies in the responses, the very purpose of this procedure is to reduce the amount of data: there may be many justice evaluations about the various income amounts assigned to a vignette, but there can be only one amount that a respondent thinks is just for a particular person. 
After removing the illogical cases and estimating the just amounts, the resulting dataset comprises a total of 38,183 observations from 1,964 respondents about fair earnings portrayed in 996 vignettes. Note that with 2,001 study participants and 21 vignettes per participant, the total number of vignette-respondent combinations is 42,021 . Thus, excluding the illogical cases reduces the sample size available for analysis by 9.1 percent.

\section{Study 2}

In Study 2, there were 2,004 participants. Again, half of the sample was female and the mean age was 41.5 years, with a standard deviation of 12.89 . With 15 vignettes per respondent, and 6 earnings amounts assigned to each vignette, the 2,004 participants in Study 2 generated 180,360 total justice evaluations. As in Study 1, we found some of the evaluations to be illogical and we decided to remove them from the analysis. We then transformed the remaining evaluations into just earnings amounts for each vignetterespondent combination, arriving at the final data set with 27,143 observations, 13,708 in Population A, and 13,435 in Population B.

\section{Hypotheses and their tests}

Our primary interest is in just gender pay gaps, which are obtained by comparing just earnings of women to just earnings of men with identical characteristics. The calculation of the just gender pay gaps proceeds in three stages, which are described in the technical appendix.

\section{Hypothesis 1}

In high-status occupations, all else being equal, the acceptable level of inequality will be higher than in low and middle positions in the occupational structure.

This hypothesis, put simply, states that gender inequality will be more tolerated in high prestige occupations compared with low and medium prestige occupations.

Figure 1 presents the results of the test of this hypothesis using results from both experiments. Note that pay gaps are expressed here as ratios on the Y-axis (values below 1 mean that women should, according to respondents, earn less than men, controlling for other relevant vignette characteristics). The estimates of just gender pay gaps are on the 
$\log$ scale. They have to be exponentiated in order to obtain the proportion of just earnings of men that women deserve. It is the latter quantities that are shown on the vertical axis of Figure 1.

Figure 1 Predicted just gender pay gaps by level of occupational prestige

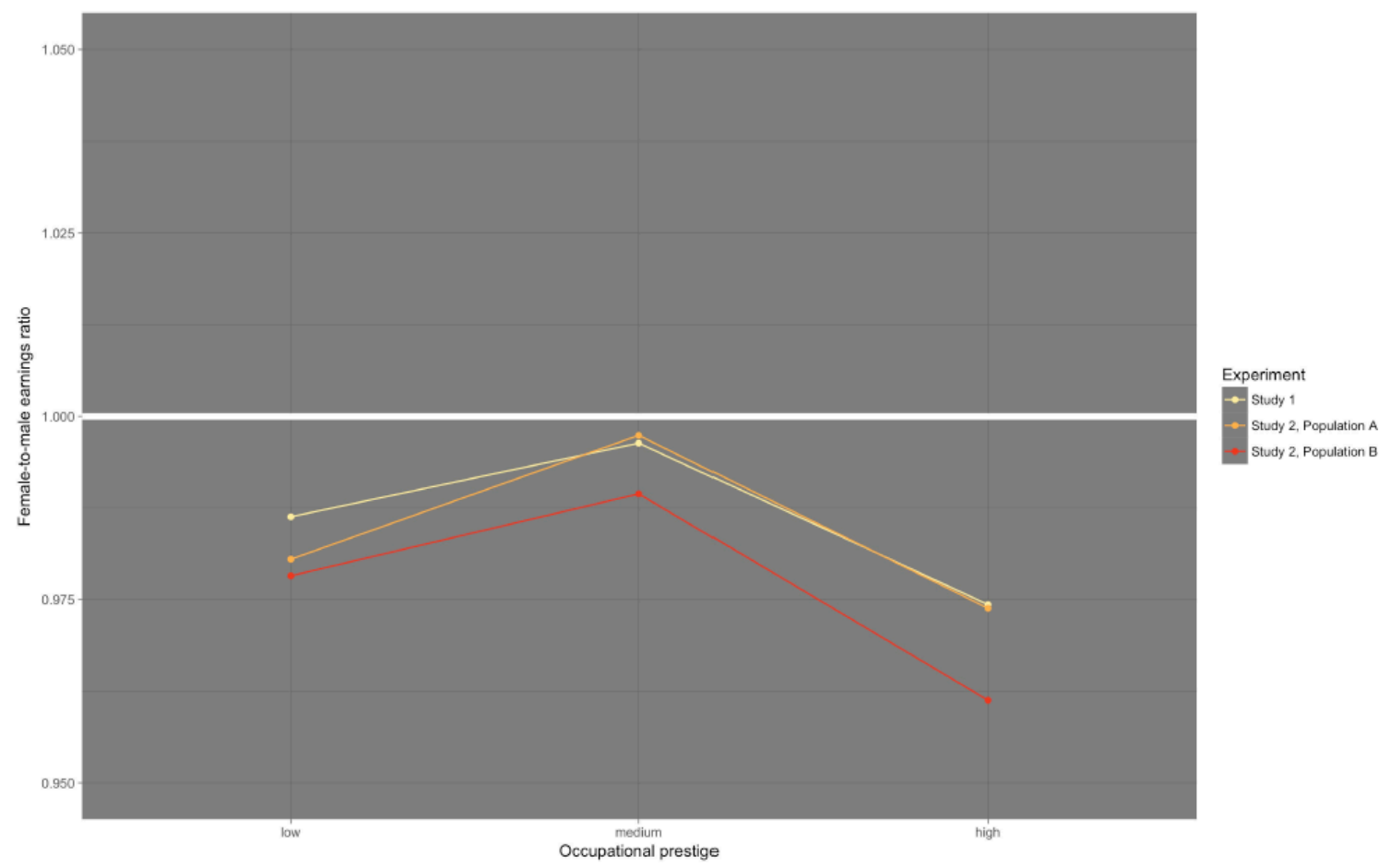

In general, what we observe is consistent with our hypothesis. That is, across the two studies and subpopulations of vignettes within study 2, accepted pay gaps are larger in high-prestige occupations than in low and medium prestige occupations, and these differences are significant.

\section{Hypothesis 2}

Ceteris paribus, being a mother in a high status occupation will be more status inconsistent than being a non-mother in a high status occupation (and, thus, the differences by prestige will be larger for mothers than for non-mothers). Being a father, on the other hand, will have little (but positive) effect on evaluations of just earnings.

Put simply this hypothesis states that the effect described in hypothesis 1 will be magnified by parenthood status. 
Figure 2 presents the results of the test of hypothesis 2 using only results from study 2 , as the subpopulation A and the population in study 1 were the same, and so are the results. Again, just pay gaps are expressed here as ratios (values below 0 mean women should earn less than men, controlling for other relevant vignette characteristics).

Figure 2 Predicted just gender pay gaps by levels of occupational prestige and the number of children

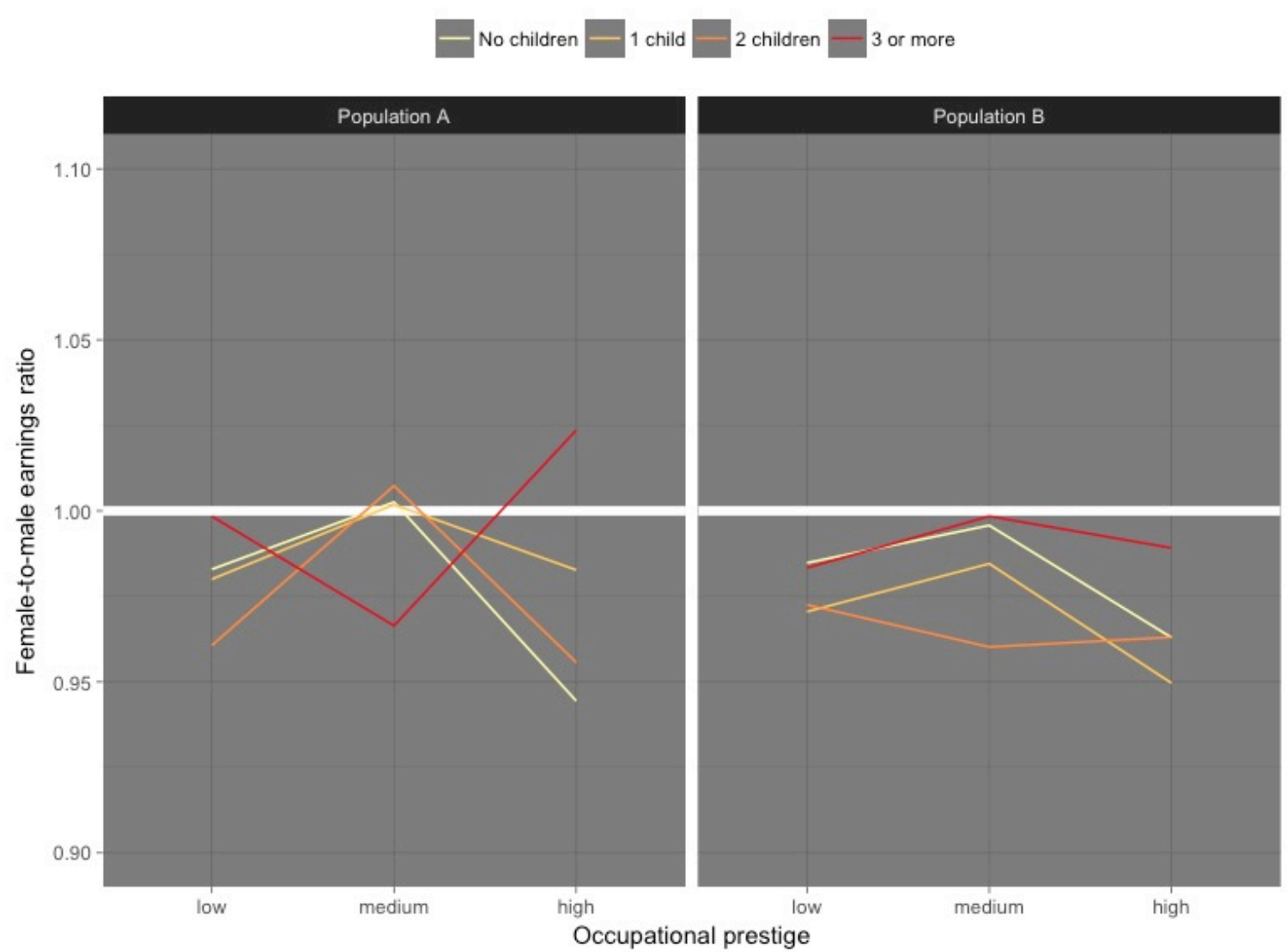

The hypothesis predicts that the prestige slope in the graphs should be steeper for mothers vs. non-mothers. Our results are not really consistent with this prediction. In general, the slopes do not vary much by parenthood status. In fact, for mothers of 3 or more children in Population A, the slope is the opposite of what we predict.

\section{Hypothesis 3 and 4}

Assuming that years of job experience constitute a specific status characteristic, we predict that acceptance of inequality of rewards for men and women will be lower for workers with more years of labor force experience compared to their less experienced counterparts. 
This effect will also be mediated by parenthood status. That is, all else being equal, additional years of experience will have a greater effect in reducing accepted pay differentials on mothers than on women with no children.

Figure 3 presents the results of the test of this hypothesis using results from study 2. As before, pay gaps are expressed here as ratios on the $\mathrm{Y}$-axis (values below 1 mean that women should earn less than men, controlling for other relevant vignette characteristics).

Figure 3 Predicted just gender pay gaps by years of on-the-job experience and the number of children

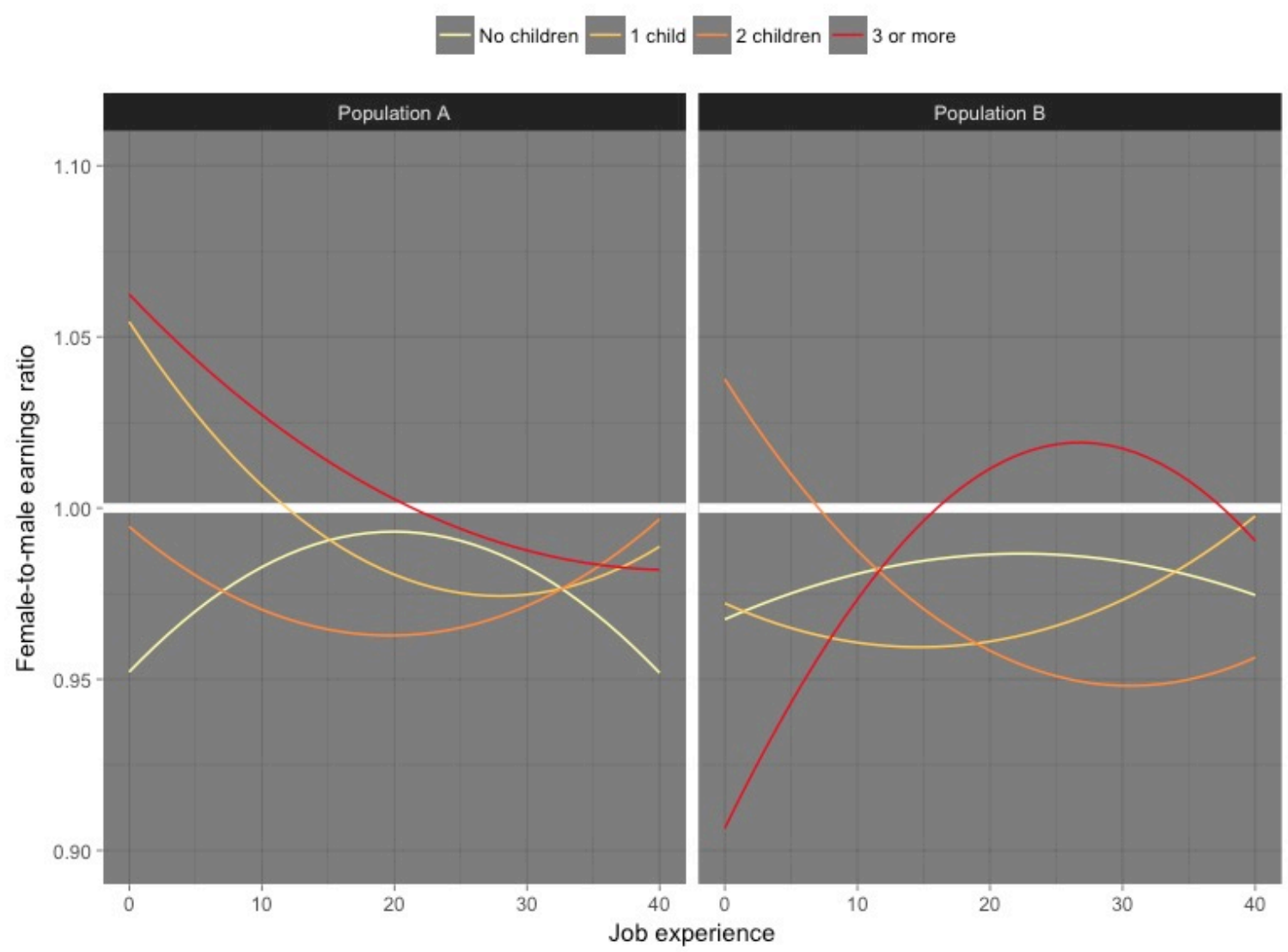

Hypothesis 3 predicts that tolerance of inequality will decrease by experience. That is, the graphs here will show an upward slope. There is visual confirmation of hypothesis 3 here (note that it is the "no-children" results that test hypothesis 3 in both sub-populations) but the results are not significant. Moreover, the slope reverses after 2025 years. 
Finally, hypothesis 4 predicts that the upward experience slope should be steeper for women with children than for non-mothers. Population A is not really appropriate for testing this hypothesis because experience here does not account for job interruptions. It is still interesting to note the situation of mothers with 3 or more children at the beginning of their careers - there is a relatively large motherhood premium.

Moving on to the more appropriate Population B graph, our results are only consistent with hypothesis 4 for parents of three or more children. The slope is steep in this case, and in fact women here reach parity with men around the 15 year mark. 


\section{Technical Appendix}

\section{Analytical strategy}

First, we transformed evaluations of the justice of earnings amounts into justice evaluations using a procedure developed by Jasso and Webster (1999; see also Jasso 2007). Underlying the procedure is an assumption that each respondent has an idea as to what each rewardee should earn, i.e., what earnings would represent just pay for each of the persons described in the vignettes. Instead of asking about just earnings directly, Jasso and Webster (1999) proposed to ask the subjects to assess a series of hypothetical amounts in terms of how fair or unfair each of these amounts would be as reward for a particular person (see, however, the discussion in Markovsky and Eriksson [2012] for a comparison of "direct" and "indirect" methods of estimating just rewards). By studying the magnitudes of injustice perceived by the respondents as a result of the assessment, we can learn how far each of the amounts departs from the "true just amount" — and in which direction. This way, we can retrieve the "true just amount" from the evaluations (see Jasso 2007; Jasso and Webster 1999 for more details). An application of the procedure to the data from our survey is described in Wysieńska-Di Carlo and Karpiński (2019).

Once the estimates of just earnings have been obtained, we proceed to look into how they vary depending on the characteristics of: $(a)$ the rewardees and; $(b)$ the respondents. That is, variation in the dependent variable - the just earnings (or, rather, their natural logarithm) is modeled here as resulting from two sources: the rewardees and the respondents. In other words, it is important to realize that the observations (i.e., the just amount) are nested both within respondents and within vignettes. To appropriately model relationships in our data, we use a mixed-effects linear model with crossed random effects of vignettes and respondents. Thus, the initial model for our data can be written as follows:

$$
\begin{gathered}
y_{g h}=\pi_{0 g}+\pi_{1} f_{h}+\pi_{2} a_{h}+\pi_{3} a_{h}^{2}+\pi_{4} l_{h}+\pi_{5} u_{h}+\pi_{6} c_{1 h}+\pi_{7} c_{2 h} \\
+\pi_{8} c_{3 h}+\epsilon_{g h}
\end{gathered}
$$




$$
\pi_{0 g}=\gamma_{00}+\sum_{k=1}^{K} \gamma_{0 k} z_{k g}+r_{0 g}
$$

In model (1), $y_{g h}$ stands for the (logged) amount of earnings that respondent $g$ beliefs are a fair pay for rewardee $h$. The quantities $f_{h}, a_{h}, a_{h}^{2}, l_{h}, u_{h}, c_{1 h}, c_{2 h}$ correspond to rewardee $h$ 's characteristics, that is, gender, age, age squared, the dummy for working in low status occupations, the dummy for working in high status occupations, the dummy for having 1 child, and the dummy for having 2 children, and the dummy for having 3 or more children, respectively. The grouped character of our data is represented by the intercept in (1a) which, as indicated by the double subscript, is allowed to vary across respondents and is modeled as depending on respondent-level characteristics, which are denoted by variables $z_{1}$ through $z_{K}$ in equation (1b). These variables are not listed here explicitly but they include, among others, gender, age, education, earnings, work situation, marital status, and the number of children. Now, our interest is in how beliefs of a particular respondent change when a particular characteristic of a given rewardee changes. That is, we are essentially interested in making within-subject comparisons, which allows us to ignore all respondent-level characteristics, because they are held constant in within comparisons. To illustrate, suppose we are comparing subject $g$ 's beliefs about just earnings of two rewardees, $h$ and $i$, who are identical in all respects except for gender: $h$ is female, while $i$ is male. Now, with gender represented as a dummy variable that is coded 1 for women, $f_{h}=1$ and $f_{i}=0$, which, after substituting to (1) gives:

$$
\begin{aligned}
y_{g h} & =\pi_{0 g}+\pi_{1}+\pi_{2} a+\pi_{3} a^{2}+\pi_{4} l+\pi_{5} u+\pi_{6} c_{1}+\pi_{7} c_{2}+\pi_{8} c_{3} \\
y_{g i} & =\pi_{0 g}+\pi_{2} a+\pi_{3} a^{2}+\pi_{4} l+\pi_{5} h+\pi_{6} c_{1}+\pi_{7} c_{2}+\pi_{8} c_{3}
\end{aligned}
$$

Note that the rewardee-level characteristics are not subscripted. This is because, as mentioned earlier, we assume that rewardees $h$ and $i$ are identical on all characteristics except for gender. Now, subtracting the latter equation from the former we get, after algebraic manipulations:

$$
y_{g h}-y_{g i}=\pi_{1}
$$


That is, $\pi_{1}$ gives us the estimate of the size of just gender gap in the eyes of respondent $g$. But, because (1) is a random intercept model in which only the intercept is allowed to vary across respondents, while all other regression coefficients are fixed, we would reach the same conclusion if we replaced $i$ with any other respondent.

\section{Gender pay gap and occupational prestige}

While the initial model in the previous section provides a useful starting point for our analysis, it is not sufficient for testing our hypotheses. The latter requires models with complex two-way and three-way interaction effects. In order to test the first hypothesis about gender and occupational prestige, we extended the initial model by adding interactions between variables representing gender and occupational prestige. This results in the following extension of the initial model:

$$
\begin{gathered}
y_{g h}=\pi_{0 g}+\pi_{1} f_{h}+\pi_{2} a_{h}+\pi_{3} a_{h}^{2}+\pi_{4} l_{h}+\pi_{5} u_{h}+\pi_{6} c_{1 h}+\pi_{7} c_{2 h} \\
+\pi_{8} c_{3 h}+\pi_{9} f_{h} \cdot l_{h}+\pi_{10} f_{h} \cdot u_{h}+\epsilon_{g h} \\
\pi_{0 g}=\gamma_{00}+\sum_{k=1}^{K} \gamma_{0 k} z_{k g}+r_{0 g}
\end{gathered}
$$

The only difference between the initial model and the previous model is the inclusion of interaction terms $f_{h} \cdot l_{h}$ and $f_{h} \cdot u_{h}$ in the former. Because of this, the predicted magnitude of the just gender pay gap will be different at different levels of occupational structure. To see this, consider two rewardees, $h$ and $i$, who work in highprestige occupations, have the same number of children, and are of the same age, but $h$ is female while $i$ is male. Thus, we have $f_{h}=1, f_{i}=0, u_{h}=1, h_{i}=1, l_{h}=0, l_{i}=0$ which, after substituting the quantities in leads to:

$$
\begin{aligned}
y_{g h} & =\pi_{0 g}+\pi_{1}+\pi_{2} a+\pi_{3} a^{2}+\pi_{5}+\pi_{6} c_{1}+\pi_{7} c_{2}+\pi_{8} c_{3}+\pi_{10} \\
y_{g i} & =\pi_{0 g}+\pi_{2} a+\pi_{3} a^{2}+\pi_{5}+\pi_{6} c_{1}+\pi_{7} c_{2}+\pi_{8} c_{3}
\end{aligned}
$$

Subtracting the latter equation from the former, we get $\pi_{1}+\pi_{10}$ as our estimate of the just gender pay gap in high-prestige occupations. This is to say that, according to the current model, just earnings for women are $\exp \left(\pi_{1}+\pi_{10}\right)$ times just earnings for men (because we use the logged amounts of just earnings in the analysis). Also, $\pi_{1}+$ 
$\pi_{10}<0$ implies that women working in high-prestige occupations are entitled to lower earnings than otherwise comparable men in the eyes of our respondents. In a similar way, the estimates of the gap at medium-prestige and low-prestige occupations are predicted to be $\pi_{1}$ and $\pi_{1}+\pi_{9}$, respectively.

However, our Hypothesis 1 claims not only that women will in general be seen as less deserving than men, but also that the magnitude of the just gender pay gap is greater in high-prestige occupations than in medium or low-prestige occupations. That is, the hypothesis predicts that

- $\quad \pi_{1}+\pi_{10}<0, \pi_{1}<0$, and $\pi_{1}+\pi_{9}<0$,

- $\pi_{1}+\pi_{10}<\pi_{1}$, which implies that $\pi_{10}<0$, and

- $\pi_{1}+\pi_{10}<\pi_{1}+\pi_{9}$, which implies that $\pi_{10}<\pi_{8}$

Table 1 Parameter estimates for the mixed-effect linear model to test Hypothesis 1 (partial output)

Dependent variable:

Log of just earnings

Study $1 \quad$ Study 2A $\quad$ Study 2B

\begin{tabular}{llll}
\hline Female & -0.004 & -0.003 & -0.011 \\
& $(0.013)$ & $(0.013)$ & $(0.014)$
\end{tabular}

Job experience

$0.009^{* * *} \quad 0.006^{* * *} \quad 0.007^{* * *}$

$(0.001)$

(0.002)

$(0.001)$

Job experience Sq.

$-0.0001^{* * *}$

$-0.0001^{* *}$

$-0.0001^{* *}$

(0.00003)

(0.00003)

(0.00004)

High prestige

$0.275^{* * *}$

$0.277^{* * *}$

$0.290^{* * *}$

(0.013)

(0.014)

(0.014)

Low prestige

$-0.126^{* * *}$

$-0.123^{* * *}$

$-0.124^{* * *}$

(0.013)

(0.013)

(0.014) 


\begin{tabular}{llll}
1 child & -0.002 & -0.002 & 0.004 \\
& $(0.009)$ & $(0.009)$ & $(0.010)$ \\
2 children & 0.013 & 0.009 & $0.032^{* * *}$ \\
& $(0.012)$ & $(0.012)$ & $(0.012)$ \\
3 or more children & $0.034^{* * *}$ & $0.033^{* * *}$ & $0.027^{* *}$ \\
& $(0.011)$ & $(0.011)$ & $(0.012)$ \\
Female|High prestige & -0.022 & -0.024 & -0.029 \\
& $(0.019)$ & $(0.019)$ & $(0.019)$ \\
Female|Low prestige & -0.010 & -0.017 & -0.011 \\
& $(0.018)$ & $(0.019)$ & $(0.019)$ \\
\hline Observations & 27,067 & 13,708 & 13,435 \\
Log Likelihood & 35,965 & 20,408 & 18,891 \\
Akaike Inf. Crit. & $-71,833$ & $-40,721$ & $-37,686$ \\
Bayesian Inf. Crit. & $-71,439$ & $-40,360$ & $-37,325$ \\
\hline Note: & ${ }^{*} \mathrm{p}^{* *} \mathrm{p}^{* * *} \mathrm{p}<0.01$ & &
\end{tabular}

The table above shows parameter estimates for the current model, fit to three datasets: the dataset from the first vignette experiment we conducted; the dataset from the second experiment, population A (see above); and the dataset from the second experiment, population B. It is important to keep in mind that the table shows only a part of the output. That is, we are showing here only the effects associated with rewardees' attributes that are directly relevant for testing Hypothesis 1 . We suppress all the other effects that we included in the model, since otherwise the resulting table would be very large and somewhat difficult to read.

The estimate of the main effect of rewardee's gender is negative and statistically insignificant at the level of $p=0.05$ across all three studies. Because gender is interacted with occupational prestige in the present model, the interpretation of the main effect of gender is limited to occupations of middle prestige. In other words, the result tells us that at the medium level of the occupational hierarchy women are not seen as deserving lower earnings than otherwise identical men. 
Let us now consider rewardees in high-prestige occupations. As demonstrated earlier, the just gender pay gap at the high level of occupational prestige is $\pi_{1}+\pi_{10}$ which, using the estimates from the first of our experiments, is equal to $-0.00366-$ $0.0224=-0.026$. The interpretation of the estimate is that participants in Study 1 view women working in high-prestige occupations as deserving $\exp (-0.026)=0.974$ of the just pay of male rewardees working in high-prestige occupations. In still other words, the just "penalty" for women working in high-prestige occupations is, in the eyes of respondents in Study 1, equal to $2.6 \%$. Just gender pay gaps at the high level of prestige in the remaining two studies can be calculated in a similar way.

Table 2 Estimates of just gender pay gaps at a high level of occupational prestige Effect Null hypothesis Estimate Std. Error $z$ statistic $p$ value

\begin{tabular}{llllll}
\hline Study 1 & $\pi_{1}+\pi_{10}=0$ & -0.026 & 0.013 & -1.94 & 0.053 \\
Study 2A & $\pi_{1}+\pi_{10}=0$ & -0.027 & 0.014 & -1.93 & 0.054 \\
Study 2B & $\pi_{1}+\pi_{10}=0$ & -0.039 & 0.014 & -2.83 & 0.005
\end{tabular}

As we can see, in all three cases, the estimate of the just gender pay gap is negative. We can also see that in the third case it is larger than in the first two. Finally, while in the first two cases the estimate is on the margin of statistical significance, in the third case it is significant.

In a similar way, we can now estimate just gender pay gaps in low prestige occupations. The results are shown below:

Table 3 Estimates of just gender pay gaps at a low level of occupational prestige

\begin{tabular}{lcclcl} 
Effect & Null hypothesis & Estimate & Std. Error & $z$ statistic & $p$ value \\
\hline Study 1 & $\pi_{1}+\pi_{9}=0$ & -0.014 & 0.013 & -1.06 & 0.287 \\
Study 2A & $\pi_{1}+\pi_{9}=0$ & -0.020 & 0.013 & -1.49 & 0.137 \\
Study 2B & $\pi_{1}+\pi_{9}=0$ & -0.022 & 0.013 & -1.64 & 0.102
\end{tabular}

Again, the estimate of the just pay gaps are negative - suggesting that women are seen as less deserving than men working in low prestige occupations - but these estimates do not reach statistical significance. In general, what we observe is consistent with our hypothesis. That is, across the two studies and subpopulations of vignettes 
within study 2, accepted pay gaps are larger in high-prestige occupations than in low and medium prestige occupations, and these differences are significant.

\section{Gender pay gap, prestige, and parenthood status}

In order to test hypothesis 2 (effect of parenthood status), we extended our statistical model even further. That is, in addition to the two-way interaction effects between gender and occupational prestige, we include in our extended model three-way interactions between gender, occupational prestige, and parenthood status (i.e., the number of children). It is precisely the three-way interaction term that is crucial for testing the present hypothesis, because it allows us to see if having children further widens the gender gap in just earnings in high prestige occupations. Put another way, using the threeway interaction term, we can see not only if women's just earnings differ from men's just earnings, but also if the gap is wider for people who have children than for people who are childless. Obviously, along with the three-way term, we add to the model all lowerlevel (i.e., two-way) interactions. The results are shown in table below. Again, we show only the subset of the output that is directly relevant for testing the hypothesis.

Table 4 Parameter estimates for the mixed-effects model to test Hypothesis 2

Dependent variable:

Log of just earnings

Study 1 Study 2A Study 2B

Female

0.002

0.003

$-0.004$

(0.023)

(0.023)

Job experience

$0.009^{* * *}$

$0.006^{* * *}$

$0.008^{* * *}$

$(0.001)$

(0.002)

(0.001)

Job experience Sq.

$-0.0001^{* * *}-0.0001^{*} \quad-0.0001^{* *}$

(0.00003)

(0.00003)

$(0.00004)$

High prestige

$0.297^{* * *}$

$0.304^{* * *}$

$0.306^{* * *}$

(0.023)

(0.023)

(0.024)

Low prestige

$-0.137^{* * *}-0.123^{* * *}-0.113^{* * *}$




\begin{tabular}{|c|c|c|c|}
\hline & $(0.023)$ & $(0.023)$ & $(0.023)$ \\
\hline \multirow[t]{2}{*}{1 child } & -0.001 & 0.0004 & 0.021 \\
\hline & $(0.023)$ & $(0.023)$ & $(0.023)$ \\
\hline \multirow[t]{2}{*}{2 children } & 0.015 & 0.0002 & $0.075^{*}$ \\
\hline & $(0.030)$ & $(0.028)$ & $(0.039)$ \\
\hline \multirow[t]{2}{*}{3 or more children } & $0.056^{* *}$ & $0.070^{* *}$ & 0.036 \\
\hline & $(0.026)$ & $(0.029)$ & $(0.025)$ \\
\hline \multirow[t]{2}{*}{ Female|High prestige } & -0.043 & $-0.060^{*}$ & -0.033 \\
\hline & $(0.032)$ & $(0.033)$ & $(0.034)$ \\
\hline \multirow[t]{2}{*}{ Female|Low prestige } & 0.002 & -0.020 & -0.011 \\
\hline & $(0.032)$ & $(0.033)$ & $(0.033)$ \\
\hline \multirow[t]{2}{*}{ Female $\mid 1$ child } & 0.0001 & -0.001 & -0.011 \\
\hline & $(0.032)$ & $(0.033)$ & $(0.033)$ \\
\hline \multirow[t]{2}{*}{ Female $\mid 2$ children } & -0.004 & 0.005 & -0.036 \\
\hline & $(0.042)$ & $(0.040)$ & $(0.047)$ \\
\hline \multirow[t]{2}{*}{ Female $\mid 3$ or more children } & -0.025 & -0.037 & 0.003 \\
\hline & $(0.037)$ & $(0.040)$ & $(0.039)$ \\
\hline \multirow[t]{2}{*}{ High prestige| 1 child } & -0.016 & -0.030 & -0.023 \\
\hline & $(0.032)$ & $(0.033)$ & $(0.034)$ \\
\hline \multirow[t]{2}{*}{ High prestige 2 children } & 0.023 & 0.005 & -0.010 \\
\hline & $(0.032)$ & $(0.033)$ & $(0.033)$ \\
\hline \multirow[t]{2}{*}{ High prestige $\mid 3$ or more children } & -0.028 & 0.002 & -0.038 \\
\hline & $(0.043)$ & $(0.043)$ & $(0.047)$ \\
\hline \multirow[t]{2}{*}{ Low prestige $\mid 1$ child } & 0.023 & 0.030 & -0.058 \\
\hline & $(0.043)$ & $(0.039)$ & $(0.047)$ \\
\hline Low prestige 2 children & $-0.068^{*}$ & $-0.090^{* *}$ & -0.041 \\
\hline
\end{tabular}




\begin{tabular}{|c|c|c|c|}
\hline & $(0.037)$ & $(0.039)$ & $(0.042)$ \\
\hline \multirow[t]{2}{*}{ Low prestige 3 or more children } & 0.001 & -0.045 & -0.003 \\
\hline & $(0.036)$ & $(0.041)$ & $(0.039)$ \\
\hline \multirow[t]{2}{*}{ Female|High prestige| 1 child } & 0.006 & 0.040 & -0.003 \\
\hline & $(0.046)$ & $(0.047)$ & $(0.048)$ \\
\hline \multirow[t]{2}{*}{ Female $\mid$ High prestige $\mid 2$ children } & -0.028 & -0.002 & -0.003 \\
\hline & $(0.045)$ & $(0.046)$ & $(0.047)$ \\
\hline \multirow[t]{2}{*}{ Female $\mid$ High prestige $\mid 3$ or more children } & 0.029 & 0.007 & 0.036 \\
\hline & $(0.059)$ & $(0.059)$ & $(0.062)$ \\
\hline \multirow[t]{2}{*}{ Female|Low prestige $\mid 1$ child } & -0.022 & -0.028 & 0.024 \\
\hline & $(0.058)$ & $(0.057)$ & $(0.062)$ \\
\hline \multirow[t]{2}{*}{ Female|Low prestige $\mid 2$ children } & 0.073 & $0.117^{* *}$ & 0.024 \\
\hline & $(0.055)$ & $(0.057)$ & $(0.061)$ \\
\hline \multirow[t]{2}{*}{ Female|Low prestige $\mid 3$ or more children } & -0.001 & 0.052 & -0.004 \\
\hline & $(0.054)$ & $(0.056)$ & $(0.057)$ \\
\hline Observations & 27,067 & 13,708 & 13,435 \\
\hline Log Likelihood & 35,928 & 20,374 & 18,855 \\
\hline Akaike Inf. Crit. & $-71,730$ & $-40,622$ & $-37,584$ \\
\hline Bayesian Inf. Crit. & $-71,213$ & $-40,148$ & $-37,112$ \\
\hline
\end{tabular}

As before, we are using linear combinations of estimated regression coefficients to test Hypothesis 2 about the effect on parenthood status on the just gender pay gap at different levels of occupational prestige. Once again, let us start by setting up the regression formula corresponding to the hypothesis: 


$$
\begin{aligned}
y_{g h}=\pi_{0 g}+ & \pi_{1} f_{h}+\pi_{2} a_{h}+\pi_{3} a_{h}^{2}+\pi_{4} l_{h}+\pi_{5} u_{h}+\pi_{6} c_{1 h}+\pi_{7} c_{2 h}+\pi_{8} c_{3 h}+\pi_{9} f_{h} l_{h} \\
& +\pi_{10} f_{h} u_{h}+\pi_{11} f_{h} c_{1 h}+\pi_{12} f_{h} c_{2 h}+\pi_{13} f_{h} c_{3 h}+\pi_{14} u_{h} c_{1 h}+\pi_{15} u_{h} c_{2 h} \\
& +\pi_{16} u_{h} c_{3 h}+\pi_{17} l_{h} c_{1 h}+\pi_{18} l_{h} c_{2 h}+\pi_{19} l_{h} c_{3 h}+\pi_{20} f_{h} u_{h} c_{1 h} \\
& +\pi_{21} f_{h} u_{h} c_{2 h}+\pi_{22} f_{h} u_{h} c_{3 h}+\pi_{23} f_{h} l_{h} c_{1 h}+\pi_{24} f_{h} l_{h} c_{2 h}+\pi_{25} f_{h} l_{h} c_{3 h} \\
& +\epsilon_{g h}
\end{aligned}
$$

where, as previously, $\pi_{0 g}=\gamma_{00}+\sum_{k=1}^{K} \gamma_{0 k} z_{k g}+r_{0 g}$ is a respondent-specific intercept term in the regression equation. Now, this formula looks a little complex but it does not introduce any new variables except for the interactions between the variables that were described earlier. In order to determine just gender pay gaps, we shall follow the approach presented earlier. That is, we first set up an equation specifying modelpredicted just earnings of a man and then a similar equation specifying just earnings of a woman with the same characteristics. In the table below, we show model predicted just gender pays gaps (or rather their logs) for each combination of the number of children and occupational prestige.

Table 5 Predicted just gender pay gaps for each combination of parenthood status and occupational prestige

The number of

children

No children

1 child

2 children

3 or more children
Low prestige

$\pi_{1}+\pi_{9}$

$\pi_{1}+\pi_{9}+\pi_{11}$

$$
+\pi_{23}
$$

$$
\begin{aligned}
& \pi_{1}+\pi_{9}+\pi_{12} \\
& +\pi_{24}
\end{aligned}
$$

$$
\begin{aligned}
& \pi_{1}+\pi_{9}+\pi_{13} \\
& +\pi_{25}
\end{aligned}
$$

Medium prestige

High prestige

$\begin{array}{cc}\pi_{1} & \pi_{1}+\pi_{10} \\ \pi_{1}+\pi_{11} & \pi_{1}+\pi_{10}+\pi_{11} \\ \pi_{1}+\pi_{12} & \pi_{1}+\pi_{10}+\pi_{12} \\ & +\pi_{21} \\ \pi_{1}+\pi_{13} & \pi_{1}+\pi_{10}+\pi_{13} \\ & +\pi_{22}\end{array}$

\section{Gender pay gap and job experience}

As stated in the main text, Hypotheses 3 and 4 refer to the effect of job experience on the acceptance of gender pay gaps. Specifically, the hypotheses predict that the accepted pay gap should decline as rewardees acquire job experience and that the effect should be 
stronger for rewardees with children. In order to test these hypotheses, we proceed as above, starting with the following mixed-effects linear model:

$$
\begin{gathered}
y_{g h}=\pi_{0 g}+\pi_{1} f_{h}+\pi_{2} e_{h}+\pi_{3} e_{h}^{2}+\pi_{4} l_{h}+\pi_{5} u_{h}+\pi_{6} c_{1 h}+\pi_{7} c_{2 h} \\
+\pi_{8} c_{3 h}+\pi_{9} f_{h} e_{h}+\pi_{10} f_{h} e_{h}^{2}+\pi_{11} c_{1 h} f_{h}+\pi_{12} c_{2 h} f_{h} \\
+\pi_{13} c_{3 h} f_{h}+\pi_{14} c_{1 h} e_{h}+\pi_{15} c_{2 h} e_{h}+\pi_{16} c_{3 h} e_{h} \\
+\pi_{17} c_{1 h} e_{h}^{2}+\pi_{18} c_{2 h} e_{h}^{2}+\pi_{19} c_{3 h} e_{h}^{2}+\pi_{20} c_{1 h} f_{h} e_{h} \\
+\pi_{21} c_{2 h} e_{h}+\pi_{22} c_{2 h} f_{h} e_{h}+\pi_{23} c_{3 h} f_{h} e_{h} \\
+\pi_{24} c_{1 h} f_{h} e_{h}^{2}+\pi_{25} c_{2 h} f_{h} e_{h}^{2}+\pi_{26} c_{3 h} f_{h} e_{h}^{2}+\epsilon_{g h} \\
\pi_{0 g}=\gamma_{00}+\sum_{k=1}^{K} \gamma_{0 k} z_{k g}+r_{0 g}
\end{gathered}
$$

The results of fitting this model to the data from our vignette experiments are shown in the table below.

Table 6 Parameter estimates for the mixed-effects linear model to test Hypothesis 3 and 4 (partial output)

Dependent variable:

Log of just earnings

Study $1 \quad$ Study $2 \mathrm{~A} \quad$ Study $2 \mathrm{~B}$

\begin{tabular}{llll}
\hline Female & 0.043 & -0.049 & -0.033 \\
& $(0.045)$ & $(0.065)$ & $(0.043)$ \\
Job experience & $0.012^{* * *}$ & 0.002 & $0.007^{* *}$ \\
& $(0.003)$ & $(0.004)$ & $(0.004)$ \\
Job experience Sq. & $-0.0002^{* *}$ & 0.00003 & -0.0001 \\
& $(0.0001)$ & $(0.0001)$ & $(0.0001)$ \\
High prestige & $0.263^{* * *}$ & $0.268^{* * *}$ & $0.275^{* * *}$ \\
& $(0.009)$ & $(0.010)$ & $(0.010)$
\end{tabular}

Low prestige

$-0.132^{* * *}-0.129^{* * *}$

$-0.129^{* * *}$ 


\begin{tabular}{|c|c|c|c|}
\hline & $(0.009)$ & $(0.009)$ & $(0.010)$ \\
\hline \multirow[t]{2}{*}{1 child } & 0.014 & -0.076 & 0.008 \\
\hline & $(0.044)$ & $(0.066)$ & $(0.044)$ \\
\hline \multirow[t]{2}{*}{2 children } & 0.027 & 0.007 & 0.040 \\
\hline & $(0.061)$ & $(0.079)$ & $(0.060)$ \\
\hline \multirow[t]{2}{*}{3 or more children } & $0.084^{*}$ & $-0.179^{* *}$ & 0.063 \\
\hline & $(0.048)$ & $(0.084)$ & $(0.052)$ \\
\hline \multirow[t]{2}{*}{ Female|Job experience } & -0.007 & 0.004 & 0.002 \\
\hline & $(0.005)$ & $(0.006)$ & $(0.005)$ \\
\hline \multirow[t]{2}{*}{ Female|Job experience sq. } & 0.0002 & -0.0001 & -0.00004 \\
\hline & $(0.0001)$ & $(0.0001)$ & $(0.0001)$ \\
\hline \multirow[t]{2}{*}{ Female $\mid 1$ child } & -0.012 & 0.102 & 0.005 \\
\hline & $(0.063)$ & $(0.092)$ & $(0.061)$ \\
\hline \multirow[t]{2}{*}{ Female $\mid 2$ children } & -0.062 & 0.044 & 0.070 \\
\hline & $(0.080)$ & $(0.120)$ & $(0.081)$ \\
\hline \multirow[t]{2}{*}{ Female $\mid 3$ or more children } & -0.062 & 0.110 & -0.065 \\
\hline & $(0.076)$ & $(0.113)$ & $(0.075)$ \\
\hline \multirow[t]{2}{*}{ Job exp.|1 child } & -0.001 & 0.007 & 0.001 \\
\hline & $(0.005)$ & $(0.006)$ & $(0.005)$ \\
\hline \multirow[t]{2}{*}{ Job exp.|2 children } & -0.001 & 0.0002 & -0.001 \\
\hline & $(0.007)$ & $(0.007)$ & $(0.007)$ \\
\hline \multirow[t]{2}{*}{ Job exp.|3 or more children } & -0.005 & $0.019^{* * *}$ & -0.002 \\
\hline & $(0.005)$ & $(0.007)$ & $(0.006)$ \\
\hline \multirow[t]{2}{*}{ Job exp. sq. $\mid 1$ child } & 0.00001 & -0.0001 & -0.00005 \\
\hline & $(0.0001)$ & $(0.0001)$ & $(0.0001)$ \\
\hline Job exp. sq. $\mid 2$ children & -0.00000 & -0.00000 & 0.00002 \\
\hline
\end{tabular}




\begin{tabular}{|c|c|c|c|}
\hline & $(0.0002)$ & $(0.0001)$ & $(0.0002)$ \\
\hline \multirow[t]{2}{*}{ Job exp. sq. $\mid 3$ or more children } & 0.0001 & $-0.0004^{* * *}$ & -0.00001 \\
\hline & $(0.0001)$ & $(0.0001)$ & $(0.0001)$ \\
\hline \multirow[t]{2}{*}{ Job exp.|1 child|Female } & 0.001 & -0.010 & -0.004 \\
\hline & $(0.007)$ & $(0.008)$ & $(0.007)$ \\
\hline \multirow[t]{2}{*}{ Job exp. $\mid 2$ children|Female } & 0.009 & -0.008 & -0.008 \\
\hline & $(0.009)$ & $(0.011)$ & $(0.009)$ \\
\hline \multirow[t]{2}{*}{ Job exp.|3 or more children|Female } & 0.005 & -0.008 & 0.007 \\
\hline & $(0.008)$ & $(0.010)$ & $(0.009)$ \\
\hline \multirow[t]{2}{*}{ Job exp. sq.|1 child|Female } & -0.00003 & 0.0002 & 0.0001 \\
\hline & $(0.0002)$ & $(0.0002)$ & $(0.0002)$ \\
\hline \multirow[t]{2}{*}{ Job exp. sq.|2 children|Female } & -0.0002 & 0.0002 & 0.0001 \\
\hline & $(0.0002)$ & $(0.0002)$ & $(0.0002)$ \\
\hline \multirow[t]{2}{*}{ Job exp. sq.|3 or more children|Female } & -0.0001 & 0.0002 & -0.0001 \\
\hline & $(0.0002)$ & $(0.0002)$ & $(0.0002)$ \\
\hline Observations & 27,067 & 13,708 & 13,435 \\
\hline Log Likelihood & 35,867 & 20,316 & 18,794 \\
\hline Akaike Inf. Crit. & $-71,608$ & $-40,506$ & $-37,463$ \\
\hline Bayesian Inf. Crit. & $-71,091$ & $-40,032$ & $-36,990$ \\
\hline
\end{tabular}

Again, this model doesn't introduce any new variables. Instead, it is an extension of our initial model (1) which includes additional effects for two-way and three-way interactions of gender, job experience (both linear and quadratic), and parenthood status. Now, proceeding as in the case of previous hypotheses, we can show that the (logged) just gender pay gaps predicted by the present model are equal to:

1. $\pi_{1}+\pi_{9} e+\pi_{10} e^{2}$ for rewardees without children

2. $\left(\pi_{1}+\pi_{11}\right)+\left(\pi_{9}+\pi_{20}\right) e+\left(\pi_{10}+\pi_{24}\right) e^{2}$ for rewardees with exactly one child, where $\pi_{11}$ is the regression coefficient for the interaction 
between dummies for being female and having one child, $\pi_{20}$ is the regression coefficient for the three-way interaction between being female, having one child, and years of job experience, while $\pi_{24}$ is the coefficient for the three-way interaction of being female, having one child, and the square of the years of schooling

3. $\left(\pi_{1}+\pi_{12}\right)+\left(\pi_{9}+\pi_{21}\right) e+\left(\pi_{10}+\pi_{25}\right) e^{2}$ for rewardees with exactly two children, where $\pi_{11}$ is the regression coefficient for the interaction between dummies for being female and having two children, $\pi_{21}$ is the regression coefficient for the three-way interaction between being female, having two children, and years of job experience, while $\pi_{25}$ is the coefficient for the three-way interaction of being female, having two children, and the square of the years of schooling

4. $\left(\pi_{1}+\pi_{13}\right)+\left(\pi_{9}+\pi_{22}\right) e+\left(\pi_{10}+\pi_{26}\right) e^{2}$ for rewardees with three or more children, where $\pi_{13}$ is the regression coefficient for the interaction between dummies for being female and having three or more children, $\pi_{22}$ is the regression coefficient for the three-way interaction between being female, having three or more children, and years of job experience, while $\pi_{26}$ is the coefficient for the three-way interaction of being female, having one child, and the square of the years of schooling

The prediction in point 1 above is used to test Hypothesis 3, while predictions in points 2 through 4 are used for testing Hypothesis 4 . 


\section{References}

Auspurg, Katrin, Thomas Hinz, and Carsten Sauer. 2017. "Why Should Women Get Less? Evidence on the Gender Pay Gap from Multifactorial Survey Experiments." American Sociological Review 82: 179-210.

Berger, Joseph, Robert Z. Norman, James W. Balkwell, and Roy F. Smith. 1992. "Status Inconsistency in Task Situations: A Test of Four Status Processing Principles.” American Sociological Review 57: 843-855.

Berger, Joseph, M. Hamit Fisek, Robert Z. Norman, and David G. Wagner. 1985. "Formation of reward expectations in status situations." In Joseph Berger and Morris Zelditch, Jr. (eds.), Status, Rewards, and Influence. San Francisco: JosseyBass.

Berger, Joseph, and Murray Webster. 2006. “Expectations, status, and behavior.” In Peter J. Burke (ed.), Contemporary Social Psychological Theories. Stanford: Stanford University Press.

Domanski, Henryk, Kazimierz M. Słomczynski, and Zbigniew Sawinski. 2009. Sociological Tools Measuring Occupations: New Classification and Scales. Warsaw: IFiS Publishers.

Foschi, Martha. 1996. "Double Standards in the Evaluation of Men and Women.” Social Psychology Quarterly 59: 237-54.

Foschi, Martha. 2000. "Double Standards for Competence: Theory and Research." Annual Review of Sociology 26: 21-42.

Heise, David R. 2010. Surveying Cultures: Discovering Shared Conceptions and Sentiments. New York: John Wiley \& Sons.

Jasso, Guillermina. 2006. "Factorial Survey Methods for Studying Beliefs and Judgements." Sociological Methods and Research 58: 334-423. 
_ 2015. "Thinking, Saying, Doing in the World of Distributive Justice." Social Justice Research 28 (4): 435-78.

Jasso, Guillermina, and Murray Webster. Jr. 1999. "Assessing the Gender Gap in Just Earnings and Its Underlying Mechanisms." Social Psychology Quarterly 62: 36780.

Jasso, Guillermina, and Murray Webster, Jr. 1997. "Double Standards in Just Earnings for Male and Female Workers." Social Psychology Quarterly 60: 66-78.

Kmec Julie A., Matt L. Huffman, and Andrew M. Penner. 2014. "Being a Parent or Having a Parent? The Perceived Employability of Men and Women Who Take Employment Leave." American Behavioral Scientist 58(3): 453- 472.

Markovsky, Barry, and Kimmo Eriksson. 2012. "Comparing Direct and Indirect Measures of Just Rewards.” Sociological Methods and Research 41: 199-216.

Ridgeway, Cecilia L., and Shelley J. Correll. 2004. "Motherhood as a Status Characteristic." Journal of Social Issues 60(4): 683-700.

Ridgeway, Cecilia L. 2001. "Inequality, Status, and the Construction of Status Beliefs." In Handbook of Sociological Theory, edited by Jonathan Turner, 323-40. New York: Springer.

Ridgeway, Cecilia L. 2006. "Status Construction Theory." In: Contemporary Social Psychological Theories, edited by Peter J. Burke, 301-23. Stanford: Stanford University Press.

Ridgeway, Cecilia L. 2014. "Why Status Matters for Inequality?” American Sociological Review 79: 1-16.

Słomczyński, Kazimierz M., and Włodzimierz Wesołowski. (2001). "Distributive justice and status (in)consistency: A theoretical debate and empirical evidence." Polish Sociological Review 135: 299-312.

Wallander, Lisa. 2009. "25 Years of Factorial Surveys in Sociology: A Review." Social Science Research 38 (3): 505-20. 
Wysieńska-Di Carlo, Kinga and Zbigniew Karpiński. 2019. "Status, Fairness, and Legitimacy of Inequality: Gender and Parenthood Effects on Perceptions of Just Incomes." SocArXiv. doi:10.31235/osf.io/f2g3t. 